\title{
CYTOLOGICAL DEMONSTRATION OF THE CLONAL NATURE OF SPLEEN COLONIES DERIVED FROM TRANS- PLANTED MOUSE MARROW CELLS
}

By Dr. A. J. BECKER, E. A. McCULLOCH and

\author{
J. E. TILL
}

Departrnent of Medical Biophysics, University of Toronto and

Ontario Cancer Institute, Toronto

IN normal mouse hæmatopoietic tissue, there is a class of cells which, on being transplanted into heavily irradiated mice, can proliferate and form macroscopic colonies. In the spleen, the colonies formed in this manner are discrete and easy to count ${ }^{1,2}$. Microscopically, each colony appears as a cluster of hæmatopoiotic cells, many of which are dividing; and often, within a given colony, the cells which are observed indicate that differentiation is occurring along three lines, into cells of the erythrocytic, granulocytic and megakaryocytic series, respectively ${ }^{1}$.

In vitro techniques, which permit investigations of clonal populations, have greatly advanced the knowledge of the genetic and physiological properties of cells ${ }^{3}$. It was therefore desirable to prove whether or not spleen colonies each develop from single cells and hence are clones; for if they are, it would be possible to study clonal populations of hæmatopoietic cells in vivo. Evidence from previous experiments supporting the view that the colonies are clones is as follows: (1) the curve relating the number of nucleated marrow cells that are transplanted to the number of colonies that develop in the spleen is linear, and shows no initial threshold ${ }^{1,2}$; (2) the radiation survival curve of cells that can form colonies closely resembles the survival curves obtained for single cells in cell culture ${ }^{4}$ or tumour transplants ${ }^{5}$. These observations provide only indirect evidence that the colonies are clones. It seemed desirable, therefore, to attempt to obtain direct evidence for the unicellular origin of the colonies.

At first glance the easiest way to accomplish this aim would be to make use of marrow cells that have a known chromosome marker, such as that found in $T 6 /+$ mice $^{6}$, and to transplant into irradiated mice a mixture of marrow cells with a marker and marrow cells with no marker. If the colonies that developed in the spleen were always composed of cells with markers or cells without markers it would, of course, suggest the single- 
cell origin of colonies; but it would not prove the single-cell origin of colonies, because the experiment would not rule out the possibility that the dispersion of donor cells was not complete and that the injected material contained undispersed aggregates of two or more cells which were the units that gave rise to colonies. The procedure used for ruling out this possibility was based on the observation of Barnes et al. ${ }^{7}$ that the re-population of the hæmatopoietic organs of heavily irradiated mice could be accomplished by a small number of cells, and that the clones originating from these few cells were sometimes characterized by unique abnormal karyotypes. This suggested that if heavily irradiated marrow were utilized for the formation of spleen colonies, some of the cells containing chromosome aberrations, generated at random by radiation, might retain the ability to proliferate and give rse to progeny carrying a recognizable, persistent chromosome abnormality. If a spleen colony develops from one such cell, it would be expected that all the cells of the colony would carry the same abnormality. If, however, a spleen colony derives from an undispersed aggregate of two or more cells, one would not expect all the cells of the colony to have the same abnormal karyotype, since the probability of inducing by radiation the same abnormality in two or more cells of an aggregate is vanishingly small.

The details of the spleen colony technique and the radiation procedures involved have been described elsewhere ${ }^{1,2}$. The particular procedure in the present experiment was to expose recipient Swiss mice of either sex to an initial X-ray dose of 250 rads. Each mouse then received an injection of $10^{7}$ nucleated bone-marrow cells, obtained from the femora of female donors of the same strain. After an interval of $1 \mathrm{~h}$, the. host mice, bearing the transplanted cells, were irradiated using a cobalt-60 $\gamma$-ray source with two doses of 325 rads each, separated by an interval of $4 \mathrm{~h}$. In this way, the transplanted cells received a total dose of 650 rads in vivo, while the host received the larger dose of 900 rads necessary to suppress the proliferation of its own colonyforming cells. As predicted from previous experiments, this radiation schedule yielded spleens containing small numbers of discrete colonies.

Eleven days later, all recipient mice wore injected intraperitoneally with 'Colcemid' (desacetymethyl colchicine, Ciba) and killed approximately $1 \mathrm{~h}$ afterwards. Their spleens were removed and individual discrete colonies were carefully dissected out. The colonies were then prepared for chromosomal analysis according to a modification of the method of Fox and Zeiss 9 .

Each colony was placed in $2 \mathrm{ml}$. of phosphate-buff6red saline which was then diluted in 4 equal steps, with distilled water, to 5 times its original volume. An interval of 10-15 min was allowed to elapse after each dilution step. The colonies were fixed for $1 \mathrm{~h}$ in a solution of 3 parts absolute alcohol and 1 part glacial acetic acid at $4^{\circ} \mathrm{C}$. They were then transferred into a solution of 40 per cent 
$\equiv$

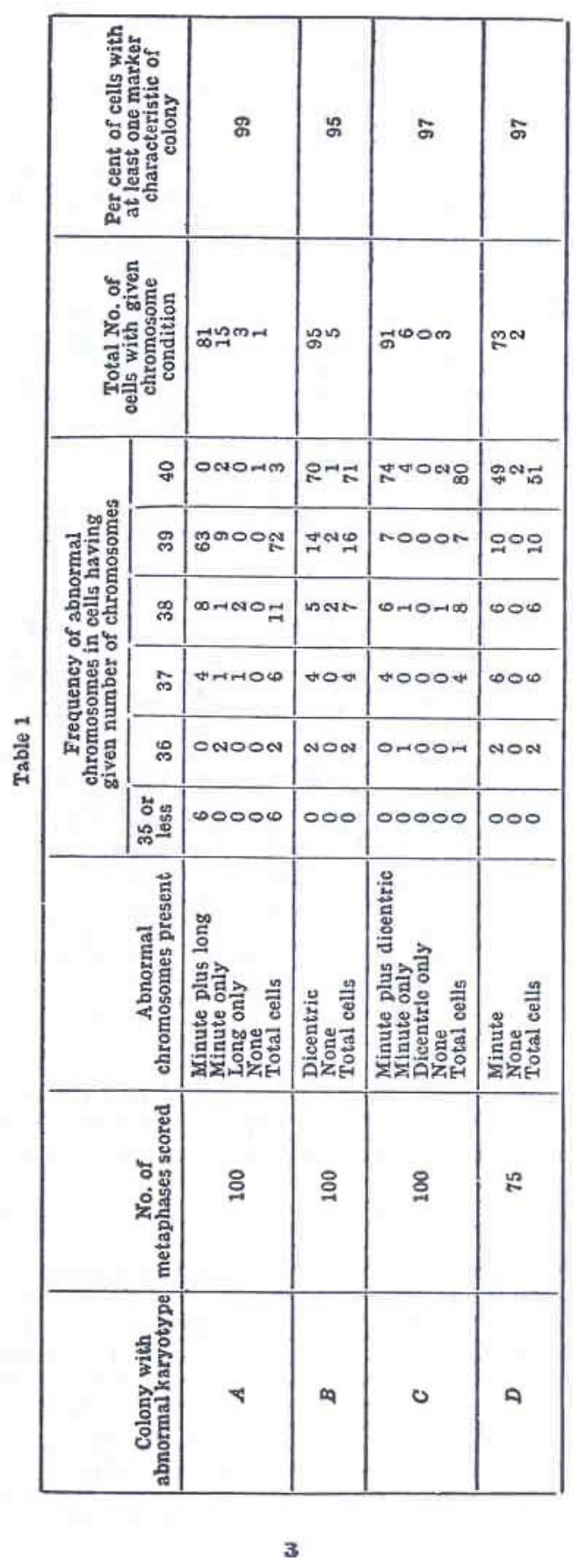




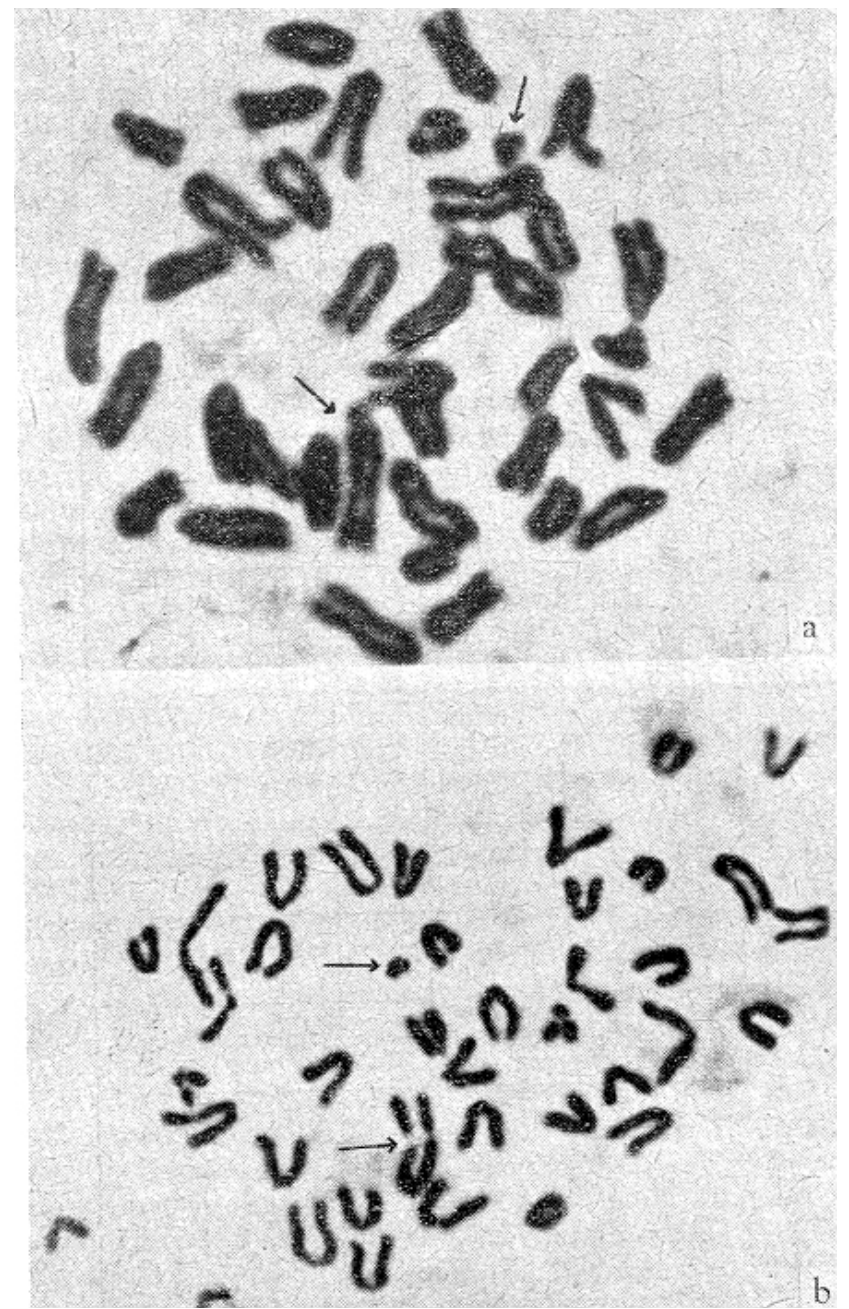

Fig. 1. Representative chromosome complements from two of the colonies listed in Table 1. The abnormal chromosomes are indicated by arrows. ( $x 1,430)$

$a$ Colony $A$, the chromosome count is 39 instead of the normal 40; $b$ colony $C$

acetic acid $\left(4^{\circ} \mathrm{C}\right)$ where they were kept for a period of $1-2 \mathrm{~h}$. Each colony was afterwards transferred into $0.5 \mathrm{ml}$. of 60 per cent acetic acid $\left(4^{\circ} \mathrm{C}\right)$ in a small plastic cup where it was teased apart by two fine needles and suspended by gentle pipetting. Small drops of this suspension were placed on the surface of a microscopic slide which had been frozen in the manner described by Fox and Zeiss ${ }^{9}$. The slide was immediately heated and dried over a gentle Bunsen flame. Staining was performed under a coverrslip with 2 per cent aceto-orcein.

Slides from each colony were systematically scanned under 100 times magnification and every metaphase cell 
which appeared sufficiently well spread without its cytoplasm being ruptured was carefully searched under oil-immersion, at 1,000 times magnification, for the presence of an abnormal karyotype. If the first 25 metaphase cells of any given colony so examined failed to reveal a readily identifiable chromosome aberration, that colony was scored as containing no obvious marker. Since female donor marrow was used, the presence, in the cells of the colonies examined, of the third unpaired small chromosome characteristic of the male karyotype ${ }^{10}$ was avoided; this facilitated identifying any odd minute chromosome encountered as a radiation-induced marker.

A total of 42 colonies obtained from 36 animals were examined in the foregoing manner. Of these colonies, 4 contained cells with an obviously abnormal karyotype. Table 1 summarizes the types of chromosome markers encountered and the frequencies of these markers within the cells of each colony. For example, mitotic figures from colony $A$ showed three characteristic abnormalities: (1) a modal chromosome number of 39; (2) an abnormally small chromosome; (3) an abnormally long sub-telocentric chromosome (Fig. 1, top). Eighty-one of 100 metaphase cells examined manifested all three abnormalities; and 99 per cent of the cells contained at least one of these distinguishing characteristics. The occasional inability to score one or the other of the two markers is probably the result of the following technical difficulties: (1) cytoplasmic rupture and chromosome loss; or (2) excessive overcrowding and overlapping of chromosomes in less-than-optimally spread metaphase cells. In the latter circumstance, whenever it was impossible to delineate with certainly one or other of the marker chromosomes, the cell was scored as lacking that particular marker. Of the 3 cells from colony $A$ which were recorded as containing 40 chromosomes, two were very difficult to count because of overcrowding of the chromosomes, and the chromosome number recorded for them may represent a miscount. Similar considerations are applicable to the interpretation of the data compiled for the other 3 marked colonies. A representative complement from colony $C$ is shown in Fig. I (bottom). It is characterized by an abnormally small chromosome and a long chromosome which is either a dicentric or has a prominent secondary constriction.

Experiments were performed to test for the presence of preexisting abnormal karyotypes among the colony-forming cells of normal donor marrow. Groups of Swiss mice were exposed to total-body doses of 900 rads; and, following irradiation, each mouse received $5 \times 10^{4}$ nucleated bone-marrow cells from female donors of' the same strain. No further irradiation was given. Of 55 colonies examined from these controls, all contained, exclusively, mitoses with the normal female diploid complement of 40 chromosomes. This finding rendered unlikely the existence, within the donor marrow, of 
uniquely and uniformly marked colouy-forming cell aggregates. The 4 abnormal karyotypes were thus the result of chromosome damage by ionizing radiation.

The results given in Table 1 show that when recognizable marker chromosomes are present in the cells of a colony, an overwhelming majority of the cells contain the same markers. Since normal mouse marrow contains no uniquely and uniformly marked colony-forming cell aggregates, and since chromosome breakage by radiation is a random process, rendering highly improbable the generation of an identical abnormality in each of the cells of a hypothetical colony-forming aggregate, it can be concluded that all the cells in these marked colonies were derived from a single cell in which a chromosome aberration was induced by radiation. Thus, in every colony where direct cytological evidence is available this evidence indicates that the colony is a clone. Because the karyotype of the mouse does not permit the identification of individual autosomes, only gross changes in the chromosomes may be identified readily. In the experiments reported here, this was possible for only approximately 10 per cent of the colonies. Nevertheless, if the direct cytological evidence is considered together with the indirect support provided by the dilution and radiation-survival data, the general view that spleen colonies are clones is a most reasonable conclusion. The spleen colony procedure may, therefore, be regarded as an in vivo single-cell technique, analogous to the well-known in vitro single-cell experimental systems ${ }^{3}$. The advantages inherent in the clonal approach to the study of cell biology are thus available for future investigations on hematopoiesis.

We thank Dr. A. A. Axelrad for his advice and Miss R. Wyncoll and Messrs. P. Csordas and J. Hicks for technical assistance. This investigation was supported by the Defence Research Board (grant 9350-14), the National Cancer Institute of Canada, and the Medical Research Council, Canada (grant MA 1282). One of us (A. J. B.) is a Fellow of the Medical Research Council, Canada.

Note added in proof. In the four additional marked colonies encountered after the submission of this article, the markers have been unique and present in all the cells.

${ }^{1}$ Till, J. E., and McCulloch, E. A., Rad. Res., 14, 213 (1961).

${ }^{2}$ McCulloch, E. A., and Till. J. E., Rad. Res., 16, 822 (1962).

${ }^{3}$ Puck, T. T., Rev. Modern Phys., 31. 433 (1959).

${ }^{4}$ Puck, T. T., and Marcus, P. I., J. Exp. Med., 103, 653 (1958)

${ }^{5}$ Hewitt, H. B., and Wilson. C. W., Brit. J. Cancer, 13, 69 (1959).

${ }^{6}$ Ford, C. E., Hamerton, J. L., Barnes, D. W. H., and Loutit, J. F., Nature, 177, 462 (1956).

${ }^{7}$ Barnes, D. W., Ford, C. E., Gray, S. M., and Loutit, J F. Prog

. Nucl. Energy (Biol.), 2. 1 (1959).

${ }^{8}$ Till, J. E., and McCulloch, E. A., Rad. Res. (in the press).

${ }^{9}$ Fox. M., and Zeiss, I. M., Nature. 192, 1213 (1961).

${ }^{10}$ Stich, H. F., and Hsu, T. C., Exp. Cell Res., 20, 248 (1960). 